\title{
Il colore nero e le sue sfumature in italiano e in polacco - un approccio contrastivo
}

\section{Black and its Hues in Italian and Polish - a Contrastive Approach}

\author{
SylwiA Skuza [sylwiaskuza@wp.pl] \\ Uniwersytet Mikołaja Kopernika w Toruniu, Polonia
}

\section{RiASSUNTO}

La ricerca è composta da tre parti. La prima descrive gli studi teorici sul colore con particolare attenzione alla teoria di B. Berlin e di P. Kay, così come ad altri significativi studi linguistici, ad esempio quelli condotti da A. Wierzbicka. Questa parte illustra anche lo stato della ricerca sul colore in entrambe le lingue d'interesse, cioè in italiano: M. Grossmann e in polacco: R. Tokarski, I. Bjelajeva. La seconda parte è principalmente incentrata sull'etimologia delle parole czarny e nero. L'ultima parte dell'articolo analizza il problema legato ai termini negro e Murzyn riferiti al colore nero della pelle umana e fino ad oggi non risolto dai linguisti in modo soddisfacente.

\section{PARole ChiAve}

nero italiano; czarny polacco; arcilessema; colore; semantica

\begin{abstract}
This paper consists of three parts. The first one describes theoretical studies on colour with particular attention paid to Berlin and Kay's theory, as well as to other significant linguistic studies, i.a. the ones conducted by A. Wierzbicka. This part also illustrates the status of research on colour in both languages of interest, i.e. Italian: M. Grossmann, and Polish: R. Tokarski, I. Bjelajeva. The second part is mostly focused on etymology of the words czarny and nero, both in Polish and Italian. Additionally, attention is paid to the Pre-Indo-European etymology defining the black colour in both Romance and Slavic languages. The last part of the article analyses describes the problems associated with the definition of people with a black skin colour (Italian negro and Polish Murzyn), which in both languages did not find a satisfactory solution.
\end{abstract}

\section{KEYWORDS}

Italian nero; Polish czarny; archilexeme; colour; semantics

RICEVUTO 2018-12-06; ACCETTATO 2019-01-25 
Il nero secondo i dizionari è un colore capace di assorbire tutte le radiazioni ottiche che riceve, in modo da non rimandarne alcuna che ecciti l'occhio. Leonardo da Vinci (1804: 72) definiva il colore nero il più scuro dei colori, sottolineando addirittura che il nero non è colore, mentre Michel Pastoureau (2008: 20) nel suo libro dedicato esclusivamente al colore nero osserva che è il colore primigenio, ma anche quello che fin dalle origini possiede uno statuto negativo: nel nero nessuna vita è possibile, la luce è buona e le tenebre no. Il nero, proprio come colore primigenio, appare non solo nella Bibbia, ma anche nella maggior parte delle mitologie europee, asiatiche e africane. Quando si tratta di raccontare o di spiegare la nascita del mondo - il nero delle origini lo troviamo sempre (Luzzatto, Pompas 2010: 45-51; Pastoureau 2008: 21-22; Zausznica 1959: 459). Bisogna anche notare che proprio il colore nero, insieme al bianco, costituisce la prima tra le categorie di colori descritte in Basic Color Terms da Brent Berlin e Paul Kay (1969). Secondo gli scienziati le lingue naturali sono caratterizzate da una terminologia cromatica che prevede da un minimo di 2 a un massimo di 12 termini. Quindi se una lingua è caratterizzata da due termini base per indicare i colori saranno per forza il nero e il bianco, se ne possiede tre saranno nero, bianco e rosso e così via.

black
white $<$ red $<$ blue $<$ brown $\quad \begin{aligned} & \begin{array}{l}\text { purple } \\ \text { pink }\end{array} \\ & \text { green } \\ & \text { orange } \\ & \text { grey }\end{aligned}$

Schema 1. Sequenza temporale di acquisizione dei termini basici ad opera di Berlin e Kay (1969: 5).

Il nero quindi è, nel suo significato primario ${ }^{1}$, il simbolo del principio; esso si presenta nelle antiche cosmogonie di tutti i popoli ${ }^{2}$ e in più il suo primato (insieme al bianco) è attestato in tutte le lingue del mondo.

\section{Etimologia e definizioni dei lessemi nero in italiano e czarny in polacco}

Il Dizionario etimologico della lingua italiana (2011: 799) indica soprattutto le origini latine del termine nero: niger, nŭgru che significava: nero, scuro, sottolineando allo stesso tempo l'incerta etimologia del lessema. Si può ipotizzare che la parola abbia le sue radici più remote nel sanscrito (Rendich 2010: 214) dato che vi troviamo il termine $n \bar{\imath}$ che fra tanti significati racchiude in sé anche la parola derivata nìla che significava blu, verde, blu scuro, nero. Questi sono i colori che le acque assumono in relazione ai cambiamenti del colore del cielo.

La conferma di questa tesi sta nelle parole di Pastoureau (2008: 28) che dice: per quanto riguarda il nero, non isola sempre completamente il suo campo cromatico da quello di altri colori (bruno, blu, viola). I ricercatori precedenti (Morselli 1882: 34) indicano le remote radici del

1 Cfr. proverbio Sopra il nero non vè colore.

2 Il nero-czarny assumeva e assume fino ad oggi anche nella cultura occidentale un ampio ventaglio di significati e delle funzioni diverse. Alla sua storia secolare Michel Pastoureau (2008), il medievalista francese, ha dedicato un libro intero. Vedi anche Rzepińska (1973), Gage (2001), Luzzato, Pompas (2010). 
lemma nero in un altro termine, proveniente anch'esso dal sanscrito: "La radice è nak e significa nero, oscuro, da cui sono derivate altre parole che conservano il significato primitivo, come il latino niger, l'italiano nero ed il sanscrito nakula, che è il nome del colore. Vicino a nak sta nif - notte e ntla - oscuro".

L'etimologia dell'aggettivo polacco $c z a r n y$ ha le sue radici nella parola proto-slava $c^{\prime}{ }^{\prime} n b^{3}$, formata dalla radice pre-indoeuropea * $\mathrm{kgs}$-no- che significava proprio nero e che a sua volta si formò dalla particella *kers che si riferiva ai colori scuri. Nella lingua polacca l'aggettivo czarny appare nel XV secolo, ancora nella forma antica czyrny come testimoniano i documenti scritti dellepoca (Boryś 2008: 90). Il significato della parola čŕn nero, ma significava pure scuro, sporco, malvagio, triste.

Nell'italiano e nel polacco moderno al termine nero si uniscono gli aggettivi vicini semanticamente di cui le nozioni indicano una qualità, un'intensità del colore nero e delle sue sfumature come scuro, buio, cupo, tenebroso. Gli aggettivi scuro-ciemny e nero-czarny, pur essendo molto simili, servono per indicare precisamente la mancanza di luce o la sua poca quantità. Il riferimento dell'aggettivo czarny - nero al fenomeno del buio completo ${ }^{4}$ è segnalato dalle prove supplementari che si riferiscono al grado d'intensità del buio, così il campo semantico dei derivati, sia dell'aggettivo italiano nero che dell'aggettivo polacco czarny, è abbastanza ampio e sviluppato.

\begin{tabular}{|c|c|c|c|c|c|}
\hline \multicolumn{6}{|c|}{$\begin{array}{l}\text { czarny } \\
\text { (nero) }\end{array}$} \\
\hline $\begin{array}{c}\mid \\
\text { czernić się } \\
\text { (annerirsi) }\end{array}$ & $\begin{array}{c} \\
\text { czernieć } \\
\text { (nereggiare) }\end{array}$ & $\begin{array}{c}\mid \\
\text { czarność } \\
\text { (nerezza) }\end{array}$ & $\begin{array}{c}\mid \\
\text { czerń } \\
\text { (nerezza) }\end{array}$ & $\begin{array}{c}\mid \\
\text { czarno } \\
\text { (nero) } \\
\mid \\
\text { czarniej } \\
\text { (neramente) } \\
\mid \\
\text { najczarniej } \\
\text { erissimamente) }\end{array}$ & $\begin{array}{c}\mid \\
\text { czarniejszy } \\
\text { (più nero) } \\
\mid \\
\text { najczarniejszy } \\
\text { (nerissimo) }\end{array}$ \\
\hline
\end{tabular}

Schema 2. Derivati dell'aggettivo czarny secondo lo schema di Dyszak (2010: 70).

3 Le parole della lingua slava comune sono ereditate dal tempo dell'unità linguistica delle tribù slave che risale al VI secolo d.C circa. Dal protoslavo čŕn ogni lingua slava ha sviluppato lessema simile per indicare il colore nero: černý nella lingua ceca, czer, czéren in bulgaro, čŕn in sloveno, czórnyj in russo ecc.

4 Cfr. proverbio italiano: Il nero non piglia altro colore. 
Le definizioni di nero e di czarny nei dizionari sono:

\begin{tabular}{|l|l|}
\hline \multicolumn{1}{|c|}{ Nero } & \multicolumn{1}{|c|}{ Czarny } \\
\hline $\begin{array}{l}\text { Dal colore più scuro, prodotto dalla assenza di luce } \\
\text { o dall'assorbimento completo dei raggi luminosi. Del } \\
\text { colore delle tenebre; oscuro (Pittano 1995). }\end{array}$ & $\begin{array}{l}\text { Mający barwę właściwą węglowi, sadzom, odpowia- } \\
\text { dającym brakom odbitych promieni świetlnych. Che } \\
\text { ha il colore del carbone, della fuliggine, il colore che } \\
\text { assorbe tutte le radiazioni visibili } \\
\text { (Szymczak 1982). }\end{array}$ \\
\hline $\begin{array}{l}\text { Di colore bruno molto scuro; del colore più scuro } \\
\text { che si conosca come quello del carbone, dellebano, } \\
\text { della notte (www.grandidizionari.it). }\end{array}$ & $\begin{array}{l}\text { Kolor najciemniejszy ze wszystkich, przeciwieństwo } \\
\text { białego. Il colore più scuro di tutti altri colori, con- } \\
\text { trapposto a bianco (Dereń, Polański 2009). }\end{array}$ \\
\hline $\begin{array}{l}\text { Detto di tutto ciò che ha un colore bruno intenso, in } \\
\text { antitesi a un equivalente chiaro } \\
\text { (Cortelazzo, Zolli 2011). }\end{array}$ & $\begin{array}{l}\text { Bardzo ciemny, najczarniejszy ze wszystkich. Molto } \\
\text { scuro, dal colore più scuro possibile (sjp.pwn.pl). }\end{array}$ \\
\hline $\begin{array}{l}\text { Scuro; talvolta per definizione o correlazione quali- } \\
\text { tativa: vino nero (contrapposto a bianco), caffè nero } \\
\text { (senza latte) (Devoto, Oli 1995) }\end{array}$ & $\begin{array}{l}\text { Mający barwę węgla, sadzy. Che ha il colore del car- } \\
\text { bone e della fuliggine (Boryś 2008: 90). }\end{array}$ \\
\hline
\end{tabular}

Tabella $^{\circ}{ }^{\circ}$. Definizioni dei termini nero e czarny.

Come possiamo notare, le definizioni italiane indicano sia il carbone che l'ebano come prototipi del colore nero, mentre quelle polacche per czarny riportano il carbone e la fuliggine come prototipi naturali e primari di questo cromonimo. Le definizioni enciclopediche del termine nero descrivono questo colore anche tramite gli aggettivi scuro, più scuro possibile o bruno intenso.

Infine bisogna osservare che le definizioni in ambedue le lingue associano univocamente il colore nero alle tenebre e all'oscurità, cioè alla notte ${ }^{5}$.

Il nero e il bianco sono decisamente i colori più spesso messi in opposizione ${ }^{6}$, il che risulta anche dalle definizioni enciclopediche in ambedue le lingue di ricerca. Per il bianco ${ }^{7}$ e il nero ci vuole

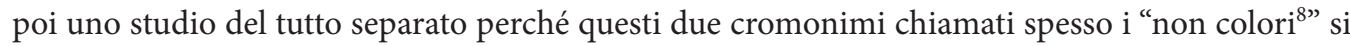
riferiscono a uno spettro molto ampio di significati opposti. La Wierzbicka (2006: 338-342) ricollega il carattere quasi-antonimico della coppia bianco-nero allopposizione tra chiaro e scuro. Nella sua opinione, la chiave per la semantica delle nozioni chiaro e scuro sta non in relazione con gli

$5 \quad$ Il motivo della notte nera è frequente anche nella poesia: Wspaniałe sa jej tuny czarną nocą. (W. Szymborska, Nienawiść). Splendidi i suoi bagliori nella notte nera. (W. Szymborska, Lodio, trad. P. Marchesani,); La neve ti poli col suo candore, i capelli ti die' la notte nera (La Calabrese, D. N. Vitale).

6 In molte espressioni idiomatiche il bianco viene spesso contrapposto al nero come il nero viene per forza contrapposto al bianco (Casadei 1996).

7 La Wierzbicka (2006: 340) sottolinea comunque che la nozione del colore bianco è molto più complicata e complessa rispetto al colore nero perché il nero ha già il suo prototipo universale $w$ smolistej czerni bardzo ciemnej nocy (nella notte nera come la pece); mentre il colore bianco non ha tale prototipo universale nel giorno chiaro perché nella luce del giorno la percezione dei colori è molto differenziata per ogni singolo individuo.

8 Molti scienziati avevano e hanno anche oggi dei dubbi circa lo status del bianco e del nero. Nel centro si pone sempre la domanda chiave se sono da considerarsi veri colori oppure no. (Deutscher 2016: 51-61). 
oggetti che vediamo ma - soprattutto - con i fenomeni atmosferici. Tale punto di vista conferma la ricerca dello studioso polacco Andrzej Dyszak (2010) dedicata alle locuzioni legate ai fenomeni riferiti al giorno (la luminosità) e alla notte (l'oscurità) e al campo semantico-lessicale delle espressioni indicanti il chiaro e lo scuro. Nonostante il rapporto antonimico rilevato dai linguisti in merito, Jurij Apresjan (1980: 381) osservò che nell'uso descrittivo dei due termini bianco - "il colore della neve" e nero - "il colore del carbone" il carattere antonimico dei lessemi viene meno. Per evidenziarlo meglio si sarebbe dovuto ricorrere a definizioni enciclopediche tipo "il colore che assorbe tutte le radiazioni visibili".

La coppia bianco-nero evoca poi tante altre coppie messe in contrasto, non solo la già nominata chiaro-scuro, ma anche notte-giorno, chiaro-buio, trasparente-opaco, pulito-sporco e persino buono-cattivo. Ryszard Tokarski (2004: 43) osserva nella lingua anche un numero rilevante di coppie degli oggetti descritti come o bianco o nero per definizione o correlazione qualitativa: caffè bianco $($ biała kawa $)=$ caffè con il latte opposto al caffè nero $($ czarna kawa $)=$ caffè senza latte; pane bianco $($ biały chleb $)=$ opposto al pane nero $($ czarny chleb $)=$ integrale ecc.

\section{Le sfumature dell'arcilessema nero in italiano e czarny in polacco}

Per esprimere la "nerezza", l'intensità della tonalità del colore, ambedue le lingue di ricerca usano espressioni che paragonano il tono del colore a un oggetto o a un animale. Gli italiani dicono nero come usando similitudini ${ }^{9}$ tipo: nero come la fuliggine, la pece, il diavolo ${ }^{10}$, il diamante, il carbone, il caffè, la notte, l'inferno, l'ebano, la morte, la grafite, l'inchiostro ${ }^{11}$. Naturalmente gli esempi dati non esauriscono l'elenco delle cose che l'uomo, per associazione paragona al colore nero. Comunque tanti fra i paragoni italiani sono identici a quelli polacchi che dicono: Czarny jak (nero come) aksamit (velluto), atrament (inchiostro), asfalt (asfalto), cygan (zingaro), diabeł (diavolo), grób (tomba), heban (ebano), kominiarz (spazzacamino), kruk (corvo), wrona (cornacchia), Murzyn (negro), noc (notte ${ }^{12}$ ), sadza (fuliggine), smok (drago), smoła (pece), święta ziemia (terra santa), węgiel (carbone).

Le sfumature del nero sia in italiano che in polacco sono piuttosto povere rispetto alle gradazioni di altri arcilessemi indicanti i colori basilari (cf. Grossmann 1988; Ampel-Rudolf 1994; Tokarski 2004; Bjelajeva 2005; Skuza 2014). Secondo Maria Grossmann (1988: 64) l'arcilessema nero possiede otto lessemi fra cui quattro - nerastro, nericcio, nerissimo, nerognolo - sono derivati ${ }^{13}$ dell'arcilessema stesso. Così rimangono altri quattro nomi di gradazioni:

9 Per descrivere il colore scuro dei capelli si usano ancora parecchi paragoni tipo: capelli neri come la pece - włosy czarne jak smoła, capelli neri come l’ebano - włosy czarne jak heban, capelli corvini - kruczoczarne włosy. Gli italiani dicono anche capelli neri come la notte che in polacco non si dice.

10 Cfr. Proverbio italiano: Il diavolo non è cosi nero come lo si dipinge. In polacco va usata di frequente la versione che sostituisce l'aggettivo czarny (nero) al posto dello straszny (orribile): Nie taki diabeł straszny, jak go malują.

11 Nero come l'inchiostro, nerissimo; nel senso figurato anche di cattivo umore.

12 Secondo Tokarski (2004: 43-44) nella descrizione del significato del colore nero-czarny si deve soprattutto cominciare dall'indicazione delle direzioni connotative del nome noc (notte) che è strettamente legato al nero, cattivo e brutto. Al nome notte aggiungiamo gli aggettivi tipo: cupa, fosca come la notte-posępny, ponury, chmurny jak noc; brutta come la notte - brzydki/a jak noc. Il nome notte-noc per la sua stretta relazione con il nero e con le tenebre viene anche associato alla morte, all'eterno riposo: noc grobowa (la notte eterna).

13 La lingua polacca dispone di una gamma abbastanza vasta degli alterati diminutivi dell'aggettivo czarny: czarnusi, czarniutki, czarnusieńki, czarnuteńki che però non sono messi dai linguisti polacchi nellelenco delle gradazioni. 


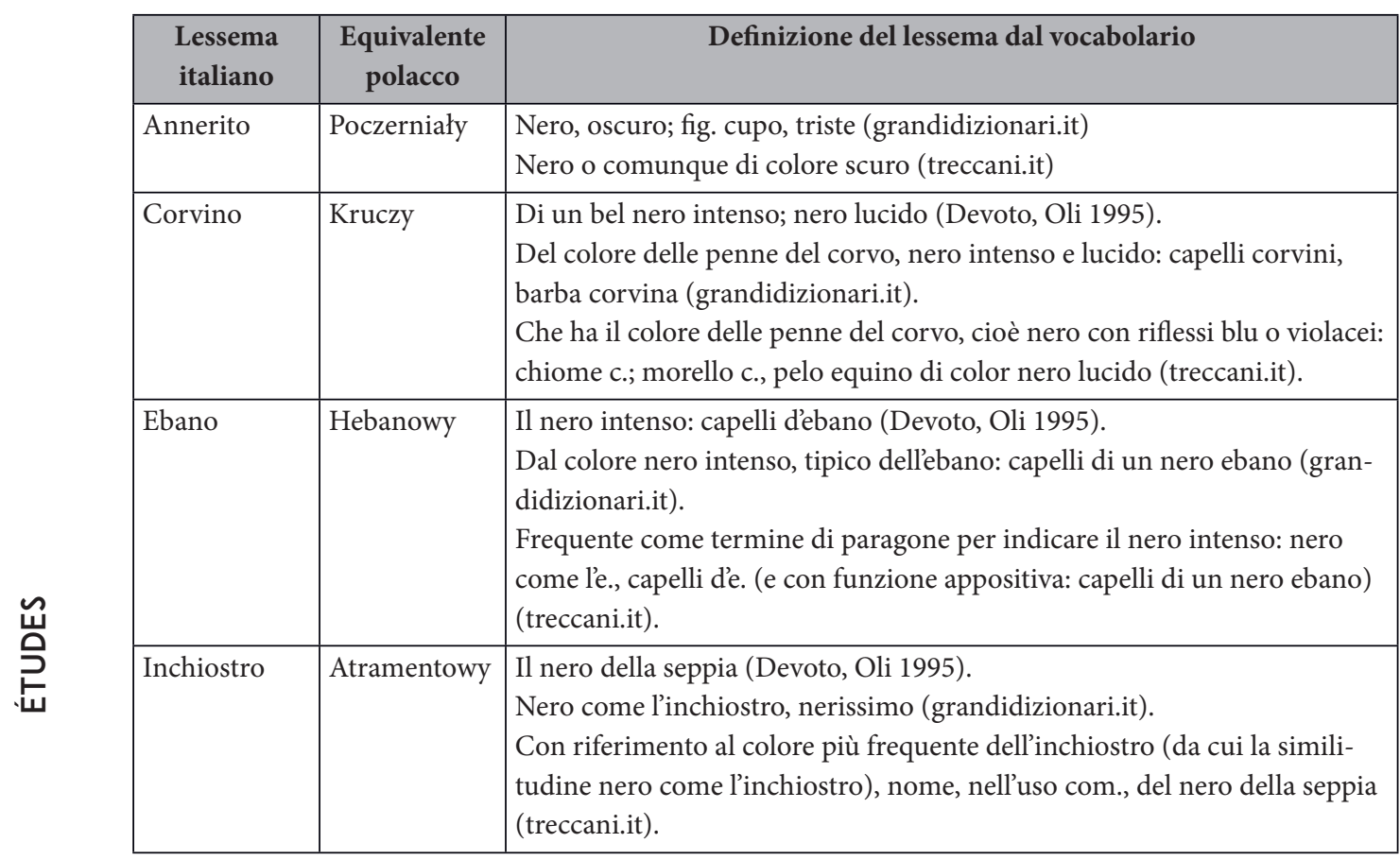

Tabella $^{\circ} 2$ Le sfumature dell'arcilessema nero.

Il lemma annerito è il derivato verbale dell'arcilessema nero, mentre altre tre gradazioni: corvino, ebano e inchiostro provengono da tre nomi (due nomi di oggetti - ebano e inchiostro- e un nome che riguarda un uccello: corvo) che si associano strettamente al colore nero.

I derivati della parola nero alludono invece alle gradazione della tonalità in generale: nerastro (czarniawy) - di colore sgradevolmente tendente al nero; nericcio (czarniawy, poczerniały) - tendente al nero (per lo più come effetto di uno sporco diffuso); nerigno (czarniawy/ciemny) - scuro (per lo più di pelle); nerognolo (czarniawy, czerniawy) - di tinta in cui il nero prevalga in modo sgradevole e disuguale.

Le tonalità del colore czarny in polacco invece sono il doppio rispetto a quelle in italiano.

\begin{tabular}{|l|l|l|}
\hline $\begin{array}{c}\text { Lessema } \\
\text { polacco }\end{array}$ & $\begin{array}{c}\text { Equivalente } \\
\text { italiano }\end{array}$ & \multicolumn{1}{c|}{ Definizione del lessema dal vocabolario } \\
\hline Antracytowy & $\begin{array}{l}\text { Antracite, co- } \\
\text { lor antracite }\end{array}$ & $\begin{array}{l}\text { Kolor szaroczarny (sjp.pwn.pl). } \\
\text { Lemma non notato (Szymczak 1982; Dereń, Polański 2009). }\end{array}$ \\
\hline Atramentowy & Inchiostro & $\begin{array}{l}\text { Ciemnogranatowy (sjp.pwn.pl). } \\
\text { Ciemny, czarny jak atrament (Dereń, Polański 2009). } \\
\text { Bardzo ciemny, czarny: atramentowa noc (Szymczak 1982). }\end{array}$ \\
\hline Grafitowy & $\begin{array}{l}\text { Nero grafite, } \\
\text { color grafite }\end{array}$ & $\begin{array}{l}\text { Kolor ciemnoszary z odcieniem srebrzystym (sjp.pwn.pl). } \\
\text { Lemma non notato (Dereń, Polański 2009). } \\
\text { Czarny z odcieniem srebrzystym (Szymczak 1982). }\end{array}$ \\
\hline
\end{tabular}




\begin{tabular}{|l|l|l|}
\hline $\begin{array}{c}\text { Lessema } \\
\text { polacco }\end{array}$ & $\begin{array}{c}\text { Equivalente } \\
\text { italiano }\end{array}$ & \multicolumn{1}{|c|}{ Definizione del lessema dal vocabolario } \\
\hline Hebanowy & $\begin{array}{l}\text { Ebano, colore } \\
\text { dellebano }\end{array}$ & $\begin{array}{l}\text { Czarny lub bardzo ciemny. (sjp.pwn.pl). } \\
\text { Mający kolor hebanu - drewna, intensywnie ciemny (Szymczak 1982; } \\
\text { Dereń, Polański 2009). }\end{array}$ \\
\hline Kary & $\begin{array}{l}\text { Morello, mo- } \\
\text { rello corvino }\end{array}$ & $\begin{array}{l}\text { O maści konia: czarny (Szymczak 1982; sjp.pwn.pl). } \\
\text { Lemma non notato (Dereń, Polański 2009). }\end{array}$ \\
\hline Kruczy & Corvino & $\begin{array}{l}\text { O włosach, piórach, sierści: intensywnie czarny i połyskliwy (Szymczak } \\
1982 ; \text { sjp.pwn.pl). } \\
\text { Intensywnie czarny i połyskliwy (Dereń, Polański 2009). }\end{array}$ \\
\hline Smolisty & Nero di pece & $\begin{array}{l}\text { Ciemny, czarny jak smoła (Szymczak 1982; sjp.pwn.pl). } \\
\text { Lemma non notato (Dereń, Polański 2009). }\end{array}$ \\
\hline Wrony & $\begin{array}{l}\text { Nero di corn- } \\
\text { acchia }\end{array}$ & $\begin{array}{l}\text { O maści konia: czarny (Szymczak 1982; sjp.pwn.pl). } \\
\text { Lemma non notato (Dereń, Polański 2009). }\end{array}$ \\
\hline
\end{tabular}

Tabella $\mathbf{n}^{\circ} 3$ Le sfumature dell’arcilessema czarny.

Le sfumature identiche del colore czarny che possiedono ambedue le lingue sono: ebano - hebanowy, inchiostro - atramentowy e corvino - kruczy. Sembra abbastanza interessante il lessema comune del nero cioè ebano-hebanowy il cui nome proviene dalla pianta delle Ebanecee che cresce nelle zone indomalesi, quindi né in Italia, né in Polonia. Il legno (di colore nero intenso) omonimo era ed è molto pregiato nella fabbricazione di strumenti musicali, mobili di lusso e oggetti ornamentali e forse per questo motivo la sua sfumatura nera si è radicata fortemente nella coscienza dei popoli europei ${ }^{14}$.

Nel caso del lemma inchiostro - atramentowy i vocabolari italiani, come vediamo, non associano questa sfumatura con il colore blu ${ }^{15}$ come è nel caso delle definizioni polacche. La Bjelajeva (2005: 49) sottolinea la cancellazione del significato primigenio di questo lessema perché originariamente l'inchiostro era nero e solo poi blu scuro, quindi solo le definizioni classiche dei dizionari che indicano atramentowy come una sfumatura di nero sono giuste. Tokarski (2004: 177-179) a sua volta spiega la tendenza dell'aggettivo atramentowy verso la sfumatura del blu scuro. Atramentowy, secondo il ricercatore, connota le associazioni legate al tramonto del sole, quando il cielo si trasforma dal blu scuro al nero; anche le acque sono spesso "contaminate" dalla sfumatura nera e così assumono un colore oscillante tra nero e blu scuro, potendo in questa situazione essere descritte proprio con l'aggettivo atramentowy.

La lingua polacca ha conservato nel campo semantico dell'arcilessema czarny due lessemi riguardanti esclusivamente il colore del manto equino. "Il Polacco ama il suo cavallo, come lo ama l'Arabo del Deserto; vive con lui, parla con lui e giunge a guidarlo in ogni verso [...]. Il

14 Sia il polacco, [per esempio nella letteratura: czarna jak heban Etiopka (Sienkiewicz 2004: 67); una etiope, nera come l'ebano (Agosti Garosci 2003: 106) che l'italiano (pelle color dell'ebano o uso fig., legno d'ebano fu nome convenzionale dei neri d'Africa nel tempo in cui si faceva la tratta per le Americhe; e mercante di ebano si diceva per eufemismo il mercante di schiavi (treccani.it)] associa il termine ebano (nome, aggettivo) al colore della pelle degli abitanti dell'Africa.

15 M. Grossmann (1988: 64) nota però nel suo lavoro la tendenza del lemma inchiostro verso la tonalità del colore blu. 
Polacco è costantemente soldato a cavallo per eccellenza" leggiamo nel Nuovo dizionario universale di agricoltura (Gera 1842: 234). Questo frammento enciclopedico dell'epoca passata spiega perché nel campo cromatico della lingua polacca si sono conservati parecchi lessemi riguardanti esclusivamente il colore del manto equino. Lanimale che fino al Novecento serviva l'uomo come mezzo di lavoro e di trasporto, in Polonia tuttora è uno degli animali più apprezzati e stimati ${ }^{16}$.

Laggettivo che riguarda il colore del manto equino kary è un prestito (del XVI secolo) dal turco, dove kara significa nero, scuro. Anticamente il lemma kary non descriveva soltanto il colore del manto equino, ma anche la pelliccia di castoro. Secondo un etimologo polacco, Wiesław Boryś (2008: 223), la parola è conosciuta nelle lingue slave dell'est, però il lavoro di Inna Bjelajeva (2005) mette in evidenza che questo lessema non fa parte del campo semantico dellarcilessema nero tranne che nella lingua polacca.

Gli aggettivi kruczy e wrony riguardano due uccelli dalle piume nere che vivono ampiamente sul territorio polacco: kruk (corvo) - kruczy e wrona (cornacchia) - wrony. Kruczy si riferisce al colore nero dei capelli che allude al nero lucente ${ }^{17}$ delle piume del corvo, mentre il lessema wrony ${ }^{18}$ riguarda esclusivamente il colore del manto equino e piano piano va in disuso.

"Nel regno vegetale incontriamo il nero nelle combustioni parziali e il carbone, che già di per sé è un corpo assai notevole, ci mostra il colore nero" (Goethe 2008: 155). Dal nome di antracite - carbone fossile paleozoico di colore nero lucente - proviene in ambedue le lingue la sfumatura chiamata antracite - antracyt. A questo punto però finiscono le similitudini in italiano e in polacco a causa della diversa classificazione relativa ai campi semantici. Antracyt secondo la Bjelajeva (2005: 48-49) e Tokarski ${ }^{19}$ (2004: 68) è il lessema appartenente all'arcilessema nero invece secondo la Grossmann (1988: 65) antracite è un iponimo del colore grigio, con la tonalità del nero. I dizionari italiani classificano decisamente antracite come tonalità che ha il colore grigio molto scuro tipico dellantracite (grandidizionari.it); una sfumatura di colore grigio piombo molto scuro (treccani. it). I dizionari polacchi invece, come poi si vede nella tabella numero 3, definiscono la gradazione antracytowy come appartenente al campo semantico del colore nero, notando allo stesso tempo la tendenza della sfumatura al colore grigio.

Nel caso dell'aggettivo polacco grafitowy - grafite (il nome grafite si riferisce al minerale costituito da carbonio puro o quasi puro, di colore nero acciaio o nero ferro) questa sfumatura del nero è raramente notata nei dizionari sia polacchi che italiani. In più le definizioni polacche, nonostante la classificazione del lessema al campo semantico del colore nero, descrivono questa sfumatura come "grigioscura con i riflessi argentei" (cf. Tabella 3). Il termine grafitowy - grafite è conosciuto comunque da tutti gli utenti della lingua, lo troviamo spesso nelle riviste illustrate, nella offerta

16 In Polonia praticamente non si consuma né si vende nei negozi la carne equina, però il Paese è paradossalmente uno dei Paesi di origine dei migliori cavalli allevati per macellazione.

17 In polacco esiste anche un aggettivo composto cioè kruczoczarny (nero corvino) che sottolinea l'intensità e la brillantezza del nero dei capelli.

18 La parola esiste nel campo semantico della lingua russa (Bjelajeva 2005: 87), ceca e slovacca (Bjelajeva 2005: 70) e ugualmente come nel caso polacco si riferisce esclusivamente al pelame equino, mentre nella lingua ucraina (Bjelajeva 2005: 102) indica generalmente una sfumatura scura.

19 Secondo il ricercatore polacco l'aggettivo antracytowy descrive una sfumatura particolare del nero, la sua eccezionale lucidità e brillantezza. 
dei diversi prodotti, descritti proprio attraverso questa sfumatura: auto in color grafite - samochód koloru grafitowego.

Le sfumature moderne per indicare le tonalità del nero sono abbastanza povere in ambedue le lingue, per questo motivo molto spesso il termine nero - czarny è accompagnato dagli aggettivi: opaco (matowy), lucido (błyszczący), profondo (głęboki), assoluto (totalny), ecc. Il settore delle tinte per i capelli propone diverse gradazioni del nero: nero blu (granatowa czerń), nero corvino (kruczoczarny), nero viola (fioletowa czerń) sia in italiano che in polacco. In ambedue le lingue notiamo anche la sfumatura asfalto-asfaltowy riferita specialmente al colore delle magliette. Le definizioni enciclopediche notano a loro volta solo la proprietà fisica (la solidità dell'asfalto) del lessema. L'italiano possiede anche il nome della tintura per scarpe nero d'inferno che in polacco non trova equivalente. Il polacco moderno usa invece a volte dei riferimenti concreti connessi con due gemme del colore nero lucido che sono l'onice nera (onyksowy) e l'ematite (hematytowy).

"Tingere in questo colore è restato a lungo un esercizio difficile e fino alla metà del XIV secolo sulla maggior parte delle stoffe e degli abiti i toni neri non sono mai veramente neri, ma presentano sempre una sfumatura grigiastra, bluastra o tendente al bruno" (Pastoureau 2008: 90). Le materie coloranti per tingere in nero venivano tratte dalla corteccia, dalle radici o dai frutti di diversi alberi: l'ontano, il noce, il castagno e alcuni tipi di querce. Verso il 1863 gli inglesi hanno elaborato il metodo di tingere di nero direttamente sulle fibre, utilizzando il cosiddetto nero di anilina (czerń anilinowa) che era il colorante più durevole e fino a oggi è di largo uso e applicazione (Trepka 1960: 111). Le vernici nere più usate nella pittura erano per secoli comunque soprattutto quelle di origine vegetale o minerale ${ }^{20}$. Nella tabella proponiamo i nomi delle sfumature delle tinte nere senza specificazione del secolo in cui sono nate o in cui godevano maggior popolarità.

\begin{tabular}{|c|c|}
\hline Il nome della tinta in italiano & Equivalente polacco \\
\hline grafite & grafit \\
\hline nero avorio, nero d'avorio & czerń z kości słoniowej, czerń słoniowa \\
\hline nero di acetilene & czerń acetylenowa \\
\hline nero di anilina & czerń anilinowa \\
\hline nero di carbonio, nerofumo & czerń węglowa \\
\hline nero d'ilmenite & czerń ilmenitowa \\
\hline nero di lampada, nero di fiamma, fuliggine & sadza \\
\hline nero di manganese & czerń manganowa \\
\hline nero d’ossa, nero animale & czerń z kości \\
\hline nero di seppia & sepia, barwnik mątwy \\
\hline nero di Spagna & czerń hiszpańska \\
\hline nero di vite & czerń z winorośli \\
\hline
\end{tabular}

20 A questo punto bisogna nominare due opere pionieristiche sulla pittura di Cennino Cennini (1370-1440) Il libro dellarte e di Leonardo Da Vinci (1452-1519) Il trattato della pittura che hanno fornito le informazioni legate sia al lessico delle vernici di allora, ma anche delle ricette come prepararli. 


\begin{tabular}{|l|l|}
\hline Il nome della tinta in italiano & Equivalente polacco \\
\hline nero minerale & czerń mineralna \\
\hline nero vegetale, carbone vegetale & węgiel roślinny, węgiel drzewny, czerń węglowa \\
\hline ossido di ferro nero & czerń żelazowa, czarny tlenek żelaza \\
\hline
\end{tabular}

Tabella $\mathbf{n}^{\circ}$ 4. L’elenco delle vernici nere in italiano e in polacco usate in pittura attraverso i secoli.

\section{Il caso dei lessemi negro e Murzyn}

Pastoureau (2008: 27) osserva che le culture antiche avevano una sensibilità più sviluppata e sfumata rispetto al colore nero (dove in tutti i campi non c'era mai solo un nero ma dei neri) di quella che mostrano le società di oggi. Il ricercatore spiega questo fenomeno con la paura della notte dei popoli della preistoria e anche con la lotta contro le tenebre unita alla permanente ricerca della luce. Ci sono due testimonianze che lo attestano: la pittura e il lessico. Noi, naturalmente, ci occuperemo della seconda spostandoci subito all'epoca dei romani dove esisteva una tavolozzza di neri ben diversificata. Laccento veniva messo soprattutto sulla testura, sulla densità e sulla brillantezza.

Il latino distingue nettamente due grandi insiemi: il nero opaco (ater) e il nero brillante (niger). Il lavoro di Maria Grossmann (1988: 106) in cui la ricercatrice indica ${ }^{21}$ trenta sei lessemi latini dell'arcilessema ater attesta proprio questa incredibile diversità delle gradazioni del nero nel mondo antico, allo stesso tempo mettendo in rilievo la povertà del vocabolario relativo alle sfumature del nero delle lingue moderne.

Nella lingua italiana non è entrato l'aggettivo ater che i dizionari latini spiegano come: nero, oscuro, triste e che già nel latino medievale diventa raro; il secondo aggettivo niger, cioè nero, scuro, fosco, funesto, malvagio, che inizialmente ha avuto solo il significato di nero brillante, con il passare del tempo è stato utilizzato per indicare tutti i neri considerati in maniera positiva e poi ha assunto su di sé la maggior parte dei significati attribuiti al nero ${ }^{22}$; per ancorarsi in maniera forte nella lingua italiana (attraverso il lemma negro), dominare molti campi semantici per poi - finalmente - perderli.

Sulla base dell'aggettivo latino niger quindi si sono formati due aggettivi italiani: negro e nero; ambedue le parole convivevano e venivano usate intercambiabilmente sulla Penisola Appenninica per esprimere la stessa cosa: il colore più scuro di tutti. Attualmente però, come risulta dalle definizioni enciclopediche, l'aggettivo negro riguarda soprattutto la razza nera e non ha quasi più niente a che fare con il colore nero ${ }^{23}$. Osserviamo che dal lemma latino niger praticamente ogni lingua romanza ha formato almeno uno e a volte anche due lessemi per indicare il colore nero: così

21 Bisogna comunque notare che questo vocabolario era instabile e impreciso.

22 Nell'Indovinello veronese, testo vergato da un ignoto copista tra l'VIII secolo e l'inizio del IX in forma d'appunto, appare proprio il lemma negro: Se pareba boves, alba pratàlia aràba et albo versòrio teneba, et negro sèmen seminaba.

23 L'uso del negro per riferirsi alle etnie di pelle scura è attestato già nella letteratura del basso medioevo (da Petrarca per es.) però il culmine del suo uso fu in epoca coloniale sia in italiano che in altre lingue (treccani.it). 
noir e nègre in francese, negru in romeno, negro in spagnolo e negro in portoghese ${ }^{24}$. Il termine negro però suscita discussioni nelle lingue romanze se riferito al colore della pelle umana (Cardeira 2016: 71-90).

Negro contemporaneamente significa: aggettivo 1. Che fa parte di una delle razze di ceppo negroide, caratterizzate da pelle scura, naso schiacciato e capelli crespi; 2. Di ciò che si riferisce o appartiene alla razza nera: letteratura, musica, pittura negra; danze negre; canti negri. Negro nome: 1. Persona appartenente a una delle razze negroidi: è un n. del Congo; la condizione dei negri; 2. scherz., gerg. Nel linguaggio editoriale e giornalistico, chi scrive testi o articoli che saranno poi firmati da altri (grandidizionari.it).

Nella lingua polacca, per chiamare una persona di pelle nera, quasi non si usava l'aggettivo nominale czarny ma per secoli (almeno dal XIV secolo) il nome Murzyn che a sua volta praticamente non usa la sua forma aggettivale. Etimologicamente la parola proviene dall'antico ceco muř́n/ múrǔnin ed è entrata nella lingua polacca come prestito durante la traduzione della Bibbia. La Sacra Scrittura veniva tradotta in polacco (XV secolo) per la prima volta dal latino, ma i traduttori si aiutavano spesso con la traduzione della Bibbia in ceco (inizio del XV secolo), così nella versione latina c'era $M_{a u r u s}{ }^{25}$, i cechi l'hanno tradotta come muřín/múřěnín che infine si trasformò in polacco nella forma Murzyn che si basava sulla pronuncia fonetica del ceco muř́n (Boryś 2008: 343). Dal nome Murzyn proviene anche l'aggettivo murzyński ${ }^{26}$ (negro) - e pure murzyć che è il verbo antico polacco oramai in disuso che significava sporcarsi di nero ma anche dire le bugie.

La forma diminutiva del nome Murzyn cioè Murzynek ogni polacco nato fino agli anni 80 del secolo scorso la associa con una poesia (del 1935) per i bimbi intitolata Murzynek Bambo (letteralmente: Negretto Bambo) del poeta polacco Julian Tuwim. La poesia, che dopo la guerra godeva di molto successo e che i piccoli polacchi recitavano a memoria, oggi si trova al centro di polemiche e da molti è considerata una poesia razzista che riflette un'immagine distorta dell'Africa.

Per tanti secoli comunque i termini negro e Murzyn non erano percepiti come termini dispregiativi, anzi, costituivano il modo più comune per riferirsi alla gente di pelle scura sia nel linguaggio comune (per esempio in polacco si diceva spesso murzyńskie wargi che tradotto in italiano sarebbe: le labbra da negro per descrivere le labbra carnose) che nella letteratura:

Dwie z nich, Murzynki, podobne do wspaniałych posąów z hebanu [...] (Sienkiewicz 1896).

Due di quelle, negre, simili a splendide statue di ebano [...] (Agosti Garosci 1928).

Come vediamo dall'esempio - sia lo scrittore polacco H. Sienkiewicz, che la traduttrice del suo romanzo C. Agosti Garosci, ancora all'inizio del XX secolo consideravano più giuste e adeguate le parole Murzynki - negre per chiamare le donne di pelle nera.

L' Accademia della Crusca ha dedicato un articolo intero all'uso dei termini negro, nero e di colore per descrivere e caratterizzare una persona, o un gruppo di persone, in base al colore della sua

24 In spagnolo prieto e in portoghese preto sono lessemi che appartengono strettammente al campo semantico dell'arcilessema negro.

25 Moro - nel senso del nome, significa abitante nativo della Mauritania, per estensione significa: Africano, Saraceno musulmano e in conseguenza - persona scura di pelle o dai capelli neri (grandidizionari.it).

26 Aggettivo murzyński significherebbe solamente un prodotto fatto da una persona di pelle nera. 
(o della loro) pelle $\mathrm{e}^{27}$ in cui risponde alle domande sulla distinzione tra nero, negro e di colore. Fino agli anni Settanta, negro, nero e di colore sono stati usati quasi come sinonimi e con connotazioni di significato molto simili (tutt'al più, erano caratterizzati da un diverso uso sintattico, essendo gli ultimi due impiegati soprattutto in funzione aggettivale). Il motivo della trasformazione della percezione del significato della parola negro è legato soprattutto all'influsso della cultura anglosassone e l'estensione alla lingua italiana e polacca della cosiddetta correttezza politica (political correctness). Nell'ambito anglosassone il nome nigger di cui l'equivalente italiano sarebbe proprio negro, è considerato molto offensivo verso una persona di pelle nera. Attualmente, per essere corretti, si preferisce sostituire la parola negro con persona di colore o nero o africano/afroamericano. L'interferenza inglese quindi ha cambiato completamente la corretta e innocente parola negro che definiva una persona originaria della cosiddetta Africa nera (in contrapposizione all'Africa Mediterranea). Comunque l'uso del termine negro in Italia senza connotazioni dispregiative ha ancora qualche diffusione e soltanto l'unione di questa parola con l'aggettivo ${ }^{28}$ sporco ha provocato ultimamente la condanna del Tribunale: dire "sporco negro 29 " è reato ${ }^{30}$.

L'espressione di colore - da molti ritenuta neutra e priva di connotazione negativa - è stata in anni recenti messa sotto accusa dall'Accademia in un intervento, alla fine del quale si evidenzia che pure il termine nero è da condannare, in quanto "non è privo di connotazioni ambigue".

Qual è quindi la scelta migliore per chiamare una persona di pelle nera? "Il punto vero, infatti, è che - al di là di opzioni più o meno accettate - sarebbe meglio specificare il colore della pelle solo se effettivamente necessario ai fini della comprensione del messaggio o dell'informazione che si vuole trasmettere" - rispondono gli accademici.

Da qualche anno anche nella lingua polacca comincia ad essere sconsigliato ${ }^{31}$ l'uso del nome Murzyn, si suggerisce di sostituirlo con le parole come czarny (nero), czarnoskóry (di pelle nera) o Afrykańczyk (africano), però i dizionari della lingua polacca insieme ai linguisti più avveduti come J. Bralczyk e J. Miodek considerano questo nome come il termine neutrale. Infatti, per secoli, fino all'accesso della Polonia nell'Unione Europea (2004), la parola Murzyn non aveva sfumature negative, ma piuttosto neutrali ${ }^{32}$. In polacco il lemma ha diversi significati dipendenti dall'uso della maiuscola o minuscola, come: Murzyn - l'uomo che appartiene alla razza ${ }^{33}$ nera; murzyn - un uomo fortemente abbronzato, qualcuno che lavora pesantemente senza essere giustamente gratificato (sjp.pwn.pl).

27 F. Faloppa, n. 43, aprile 2006, Crusca per voi, consultabile al link http://www.accademiadellacrusca.it/it/lingua-italiana/consulenza-linguistica/domande-risposte/nero-negro-colore [2018-11-12]

28 Quindi l'insulto è nell'aggettivo che può accompagnarlo oppure nel tono della voce.

29 Negro sporco nella traduzione in polacco si racchiude in una sola parola: czarnuch (ugualmente offensiva, derivata dall'aggettivo czarny).

30 Consultabile al link http://www.unita.it/italia/la-cassazione-dire-sporco-negro-br-e-reato-da-tribunale-1.273943 [2018-11-15]

31 Consultabile al link http://poradnia.pwn.pl/lista [2018-11-15]

32 Secondo il professore polacco Marek Łaziński (2007: 47-56) in futuro la parola Murzyn, pur non avendo accezioni dispregiative o razziste, verrà sostituita con il termine Czarny con motivo dell'unificazione linguistica proposta dall'UE.

33 Il termine rasa (razza) in riferimento all'uomo è accettato nella lingua polacca nonostante le controversie e le discussioni. 
I lessemi: l'italiano negro e il polacco Murzyn funzionano nelle frasi idiomatiche legate soprattutto alla crudele e dura tratta degli schiavi africani: Lavorare/sudare come un negro - robić za Murzyna o harować jak Murzyn - lavorare pesantemente.

Biały murzyn - espressione polacca tradotta come: negro bianco. Riguarda un uomo sfruttato, trattato quasi come uno schiavo, il cui lavoro è durissimo e mal pagato.

Ciemno jak w dupie u Murzyna - locuzione polacca che, in modo offensivo, descrive il buio o la nerezza dell'ambiente (tradotto letteralmente: buio come dentro al culo di un negro).

Il proverbio Murzyn zrobił swoje, murzyn może odejść viene usato in Polonia quando qualcuno ha lavorato pesantemente e il suo lavoro non è stato apprezzato né pagato.

Opalić się na murzyna - abbronzarsi molto.

\section{Conclusioni}

Le sfumature dell'arcilessema italiano nero sono assai povere rispetto al numero delle gradazioni latine. Nel caso dell'equivalente polacco czarny questo numero è un po' più alto, ma certe gradazioni, come wrony, vanno in disuso o sono molto limitate nell'uso come è nel caso del lemma kary. Possiamo notare che, anche se i riferimenti ai prototipi variano da lingua a lingua oppure nell'ambito delle realtà geo-culturali, nel caso dei lessemi nero e czarny osserviamo unanimità prototipica legata alla notte, al carbone o alla pece. Molte discussioni sul campo linguistico suscita, soprattutto in Italia, ma da qualche anno pure in Polonia, il problema della terminologia legata al colore nero della pelle umana fino ad oggi non risolto in modo soddisfacente.

\section{Riferimenti bibliografici}

Apresjan, J. (1980). Semantyka leksykalna. Synonimiczne środki języka. Wrocław: Ossolineum.

Berlin, B.; \& Kay, P. (1969). Basic Color Terms. Their Universality and Evolution. Berkeley: CSL Publications.

Bjelajeva, I. (2005). Niepodstawowe nazwy barw w języku polskim, czeskim, rosyjskim i ukraińskim. Warszawa: Wydawnictwo UW.

Boryś, W. (2008). Słownik etymologiczny języka polskiego. Kraków: Wydawnictwo Literackie.

Cardeira, E. (2016). Preto and negro, pardo, metiço and mulato. In J. P. Silvestre, \& E. Cardeira. (Ed.), Colour and colour naming: crosslinguistic approaches (pp. 71-90). Lisboa: Centro de Linguística da Universidade de Lisboa.

Casadei, F. (1996). Metafore ed espressioni idiomatiche. Uno studio semantico sullitaliano. Roma: Bulzoni Editore.

Cortelazzo, M.; \& Zolli, P. (2011). Dizionario etimologico della lingua italiana. Bologna: Zanichelli.

Da Vinci, L. (1804 [1472]). Trattato della pittura. Milano: Società Tipografica de' Classici Italiani.

Deutscher, G. (2016). La lingua colora il mondo. Come le parole deformano la realtà. Torino: Boringhieri.

Devoto, G.; \& Oli, G. (1995). Il dizionario della lingua italiana. Firenze: Le Monnier.

Dyszak, A. (2010). Językowe wyrażenia zjawisk jasności i ciemności. Bydgoszcz: Wydawnictwo UKW. 
Grossmann, M. (1998). Colori e lessico. Studi sulla struttura semantica degli aggettivi di colore in catalano, castigliano, italiano, romeno, latino e ungherese. Tübingen: Narr.

Gage, J. (2001). Colore e cultura. Usi e significati dall'antichità all'arte astratta. Roma: Editore Poligrafo dello Stato.

Gera, F. (1842). Nuovo dizionario universale di agricoltura. Vol. XIX. Venezia: Giuseppe Antonelli Editore.

Goethe, J. (2008). La teoria dei colori. Milano: Il Saggiatore.

Luzzatto, L.; \& Pompas, R. (2010). Il significato dei colori nelle civiltà antiche. Milano: Bompani.

Łaziński, M. (2007). Murzyn zrobił swoje, czy Murzyn musi odejść? Historia i przyszłość słowa Murzyn w polszczyźnie. Poradnik Językowy, 4, 47-56.

Morselli, E. A. (1882). Rivista di filosofia scientifica. Vol. 1. Torino: Fratelli Dumolard.

Pastoureau, M. (2008). Nero. Storia di un colore. Milano: Ponte Alle Grazie.

Pittano, G. (1995). Bidizionario italiano, linguistico e grammaticale. Santarcargelo di Romagna: Gulliver.

Polański, E.; \& Dereń, E. (2009). Wielki słownik języka polskiego. Kraków: PWN.

Rendich, F. (2010). Dizionario etimologico comparato delle lingue classiche indoeuropee. Roma: Palombi Editori.

Rzepińska, M. (1973). Historia koloru w dziejach malarstwa europejskiego. Kraków: Wydawnictwo Literackie.

Sienkiewicz, H. (2004 [1896]). Quo vadis? Kraków: Wydawnictwo Greg. . (2003 [1928]). Quo vadis? Trad. C. Agosti Garosci. Milano: RCS Libri.

Skuza, S. (2014). Rosso, giallo, blu. Unianalisi etnolinguistica sui colori primari in italiano e in polacco in prospettiva sincronica e diacronica. Toruń: Wydawnictwo UMK.

Szymczak, M. (1982). Słownik języka polskiego. Warszawa: PWN.

Tokarski, R. (2004). Semantyka barw we współczesnej polszczyźnie. Lublin: UMCS.

Trepka, E. (1960). Historia kolorystyki. Warszawa: PWN.

Wierzbicka, A. (2006). Semantyka. Jednostki elementarne i uniwersalne. Lublin: UMCS.

Zausznica, A. (1959). Nauka o barwie. Warszawa: PWN. 